\title{
Ultrasound-Guided T12 paravertebral block combined with L1-4 nerve roots block and sacral plexus block for hip surgery: A Case Series
}

\author{
Daqiang ZHAO | daqiang.zhao@jiahui.com \\ Yang TANG
}

T12 paravertebral block combined with L1-4 nerve roots block and sacral plexus block can completely block the motion, feeling and sympathetic nerve of the hip joint, and provide a safe and effective anesthesia method for the elderly patients who receive hip surgery.
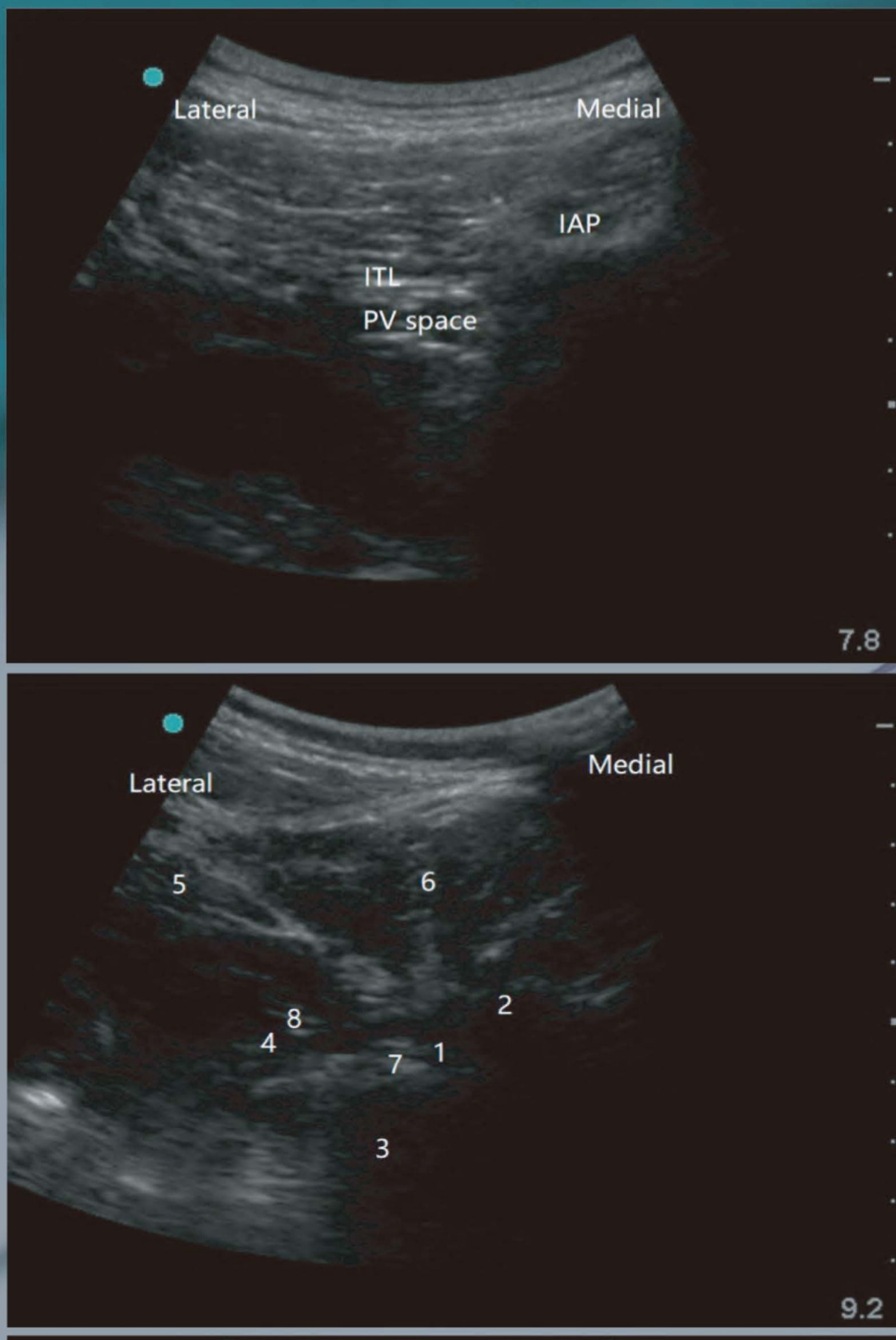

1. intervertebral foramen;

2. articular process;

3. vertebral body;

4. psoas muscle;

5. quadratus lumborum muscle;

6. erector spinae muscle;

7. lumbar nerve root;

8. lumbar plexus

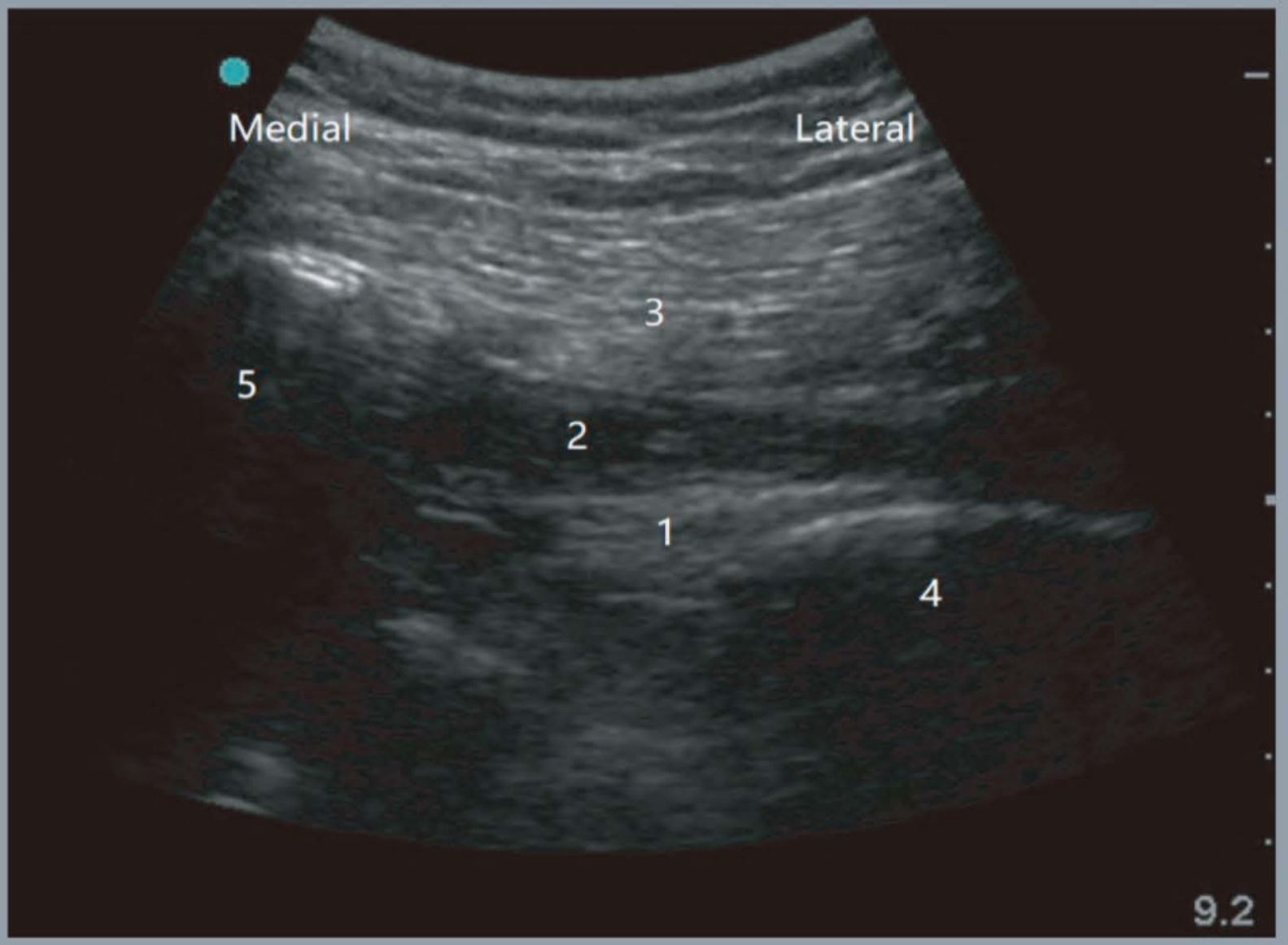

1. sacral plexus;

2. piriformis muscle;

3. gluteus maximus;

4. ilium;

5. sacrum 